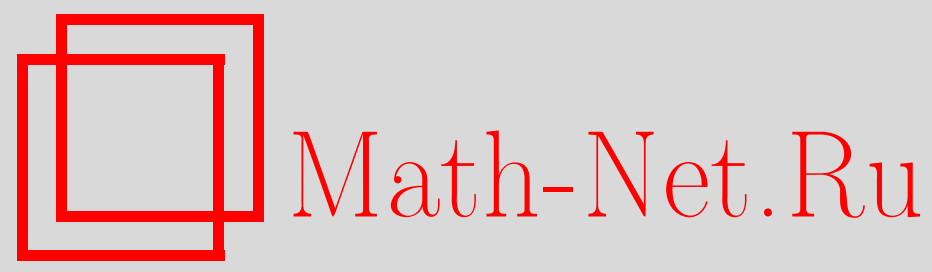

А. В. Карташова, О конечных решетках топологий коммутативных унарных алгебр, Дискрет. матем., 2009, том 21, выпуск 3, 119-131

DOI: https://doi.org/10.4213/dm1065

Использование Общероссийского математического портала Math-Net.Ru подразумевает, что вы прочитали и согласны с пользовательским соглашением http://www . mathnet.ru/rus/agreement

Параметры загрузки:

IP : 54.81 .137 .203

26 апреля 2023 г., $14: 13: 26$ 


\title{
О конечных решетках топологий коммутативных унарных алгебр
}

\author{
() 2009 г. А. В. Карташова
}

\begin{abstract}
В работе показано, что решетка топологий конечной однопорожденной коммутативной унарной алгебры изоморфна решетке топологий характеристической полугруппы этой алгебры. С использованием этого утверждения охарактеризован класс всех коммутативных унарных алгебр с линейно упорядоченными решетками топологий. Установлено, что если либо решетка конгруэнций, либо решетка топологий коммутативной унарной алгебры конечна, то и сама алгебра конечна. Приведены примеры бесконечных некоммутативных унарных алгебр с конечными решетками топологий. Доказано, что для произвольной функциональной сигнатуры, содержащей хотя бы один символ, арность которого больше 1 , и любого целого числа $n \geqslant 2$ найдется бесконечная алгебра этой сигнатуры, решетка топологий которой линейно упорядочена и состоит из $n$ элементов.
\end{abstract}

\section{1. Введение}

Унарной алгеброй называется алгебра, сигнатура которой состоит только из унитарных символов. Очевидно, что любую унарную алгебру можно рассматривать как полигон над моноидом или автомат без выхода. В силу этих обстоятельств унарные алгебры постоянно привлекали и привлекают внимание многих исследователей (см., напр., [1-6]). Значительное место в теории унарных алгебр занимают исследования решеток, связанных с алгебрами. Эти решетки несут важную информацию о свойствах самих алгебр. К ним относятся, например, наиболее изученные на сегодняшний день решетки подалгебр и конгруэнций.

В последнее время в связи с активным применением топологических методов в алгебре (см., напр., $[7,8])$ становится актуальной задача исследования решеток топологий алгебр. В настоящей работе охарактеризован класс всех коммутативных унарных алгебр с линейно упорядоченными решетками топологий. Установлено, что если решетка конгруэнций, либо решетка топологий коммутативной унарной алгебры конечна, то и сама алгебра конечна. Приведены примеры бесконечных некоммутативных унарных алгебр с конечными решетками топологий. Доказано, что для произвольной функциональной сигнатуры, содержащей хотя бы один символ, арность которого больше 1 , и любого целого числа $n \geqslant 2$ найдется бесконечная алгебра этой сигнатуры, решетка топологий которой линейно упорядочена и состоит из $n$ элементов. Некоторые из приведенных в статье результатов анонсированы в [9]. 


\section{2. Определения и вспомогательные результаты}

Пусть $\mathfrak{U}=\langle A, \Omega\rangle$ - произвольная алгебра и $\delta$ - топология на множестве $A$. Сигнатурная $n$-арная операция $F$ называется непрерывной относительно топологии $\delta$, если для любых элементов $a_{1}, a_{2}, \ldots, a_{n} \in A$ и произвольной окрестности $U$ элемента $F\left(a_{1}, a_{2}, \ldots, a_{n}\right)$ найдутся окрестности $U_{1}, U_{2}, \ldots, U_{n}$ элементов $a_{1}, a_{2}, \ldots, a_{n}$, соответственно, такие, что $F\left(U_{1}, U_{2}, \ldots, U_{n}\right) \subseteq U$. Топология на множестве $A$, относительно которой каждая операция из $\Omega$ является непрерывной, называется топологией на алгебре $\mathfrak{A}$. Множество всех топологий на алгебре $\mathfrak{A}$, упорядоченное по включению, является полной решеткой, которую будем обозначать $\mathfrak{I}(\mathfrak{U})$. Решетки подалгебр и конгруэнций алгебры $\mathfrak{A}$ обозначаются через Sub $\mathfrak{U}$ и Con $\mathfrak{A}$, соответственно. Подалгебра алгебры $\mathfrak{U}$, порожденная элементом $a \in A$, обозначается $(a)$. Далее всюду $\mathbf{Z}-$ множество всех целых чисел, $\mathbf{N}-$ множество целых положительных чисел и $\mathbf{N}_{0}=\mathbf{N} \cup\{0\}$.

Для любой унарной алгебры $\mathfrak{U}=\langle A, \Omega\rangle$ через $\Omega^{*}$ обозначается свободный моноид слов с базой $\Omega$. Единицей в $\Omega^{*}$ является пустое слово. Для любого слова $u \in \Omega^{*}$ определим отображение $\sigma_{u}$ равенством $\sigma_{u}(a)=u(a), a \in A$. Множество $\left\{\sigma_{u} \mid u \in \Omega^{*}\right\}$ образует полугруппу $S(\mathfrak{U})$ относительно композиции. Эта полугруппа называется характеристической полугруппой алгебры $\mathfrak{U}$ ([10]).

Унарная алгебра $\mathfrak{U}=\langle A, \Omega\rangle$ называется коммутативной, если $f(g(a)=g(f(a)$ для всех $f, g \in \Omega$ и $a \in A$.

Отметим, что если $\mathfrak{U}$ - коммутативная унарная алгебра, то каждое отображение $\sigma_{u}$, $u \in \Omega^{*}$, является эндоморфизмом алгебры $\mathfrak{U}$. Кроме того, очевидно, что в коммутативном случае

$$
S \mathfrak{U} \cong \Omega^{*} / \operatorname{Ann} A,
$$

где

$$
\text { Ann } A=\left\{(u, v) \mid u, v \in \Omega^{*}, u(a)=v(a) \text { для всех } a \in A\right\} .
$$

Наименьшую по включению топологию на произвольной унарной алгебре, содержащей данное подмножество $V$ носителя этой алгебры, назовем топологией, порожденной множеством $V$, и обозначим $t(V)$.

Непосредственно из определений вытекает следующее утверждение.

Лемма 1. Пусть $\mathfrak{U}=\langle A, \Omega\rangle-$ унарная алгебра $u V \subseteq A$. Тогда каждое непустое подмножество $X \subsetneq A$, открытое в топологии $t(V)$, равно объединению некоторых конечных пересечений множеств вида $u^{-1}(V)$, где $u \in \Omega^{*}$ (под $u^{-1}(V)$ понимается полный прообраз $V$ при отображении $\sigma_{u}$ ).

\section{3. Решетки топологий однопорожденных коммутативных унарных алгебр}

Известно (см. теорему 1 в [1]), что для любой однопорожденной коммутативной унарной алгебры $\mathfrak{U}=\langle A, \Omega\rangle$ справедливо равенство $|A|=|S(\mathfrak{U})|$ и $\operatorname{Con} \mathfrak{U} \cong \operatorname{Con} S(\mathfrak{U})$.

Ниже показано, что аналогичный результат имеет место для решетки топологий конечной однопорожденной коммутативной унарной алгебры.

Далее для любой топологии $\delta$ на конечном множестве $A$ через $M(x, \delta)$ будем обозначать наименьшую по включению окрестность элемента $x \in A$ в топологическом пространстве $(A, \delta)$. 
Теорема 1. Пусть $\mathfrak{U}$ - произвольная конечная однопорожденная коммутативная унарная алгебра. Тогда

$$
\mathfrak{I}(\mathfrak{U}) \cong \mathfrak{I}(S(\mathfrak{U}))
$$

Доказательство. Пусть $\mathfrak{U}=(a)$ и $\sigma_{u} \in S(\mathfrak{U})$. Для любой топологии $\delta \in \mathfrak{I}(\mathfrak{U})$ положим

$$
H\left(\sigma_{u}\right)=\left\{\sigma_{w} \in S(\mathfrak{U}) \mid w(a) \in M(u(a), \delta)\right\}
$$

Нетрудно убедиться, что совокупность подмножеств $H\left(\sigma_{u}\right), u \in \Omega^{*}$, носителя полугруппы $S(\mathfrak{U})$ образует базу некоторой топологии $\varphi(\delta)$ на этом множестве.

Из (1) непосредственно вытекает, что $M\left(\sigma_{u}, \varphi(\delta)\right)=H\left(\delta_{u}\right)$ при всех $u \in \Omega^{*}$. Покажем, что $\varphi(\delta)$ является топологией на полугруппе $S(\mathfrak{U})$. Согласно лемме 1 из [11], для этого достаточно проверить, что

$$
M\left(\sigma_{u}, \varphi(\delta)\right) M\left(\sigma_{v}, \varphi(\delta)\right) \subseteq M\left(\sigma_{u v}, \varphi(\delta)\right)
$$

для любых $u, v \in \Omega^{*}$.

Пусть $x, y \in \Omega^{*}, \sigma_{x} \in M\left(\sigma_{u}, \varphi(\delta)\right), \sigma_{y} \in M\left(\sigma_{v}, \varphi(\delta)\right)$. Из (1) вытекает, что $x(a) \in M(u(a), \delta), y(a) \in M(v(a), \delta)$. Следовательно,

$$
\begin{aligned}
& M(x(a), \delta) \subseteq M(u(a), \delta), \\
& M(y(a), \delta) \subseteq M(v(a), \delta) .
\end{aligned}
$$

Отсюда, в силу (3) и леммы 1 из [11], следует, что

$$
x y(a)=y x(a) \in y(M(u(a), \delta)) \subseteq M(y u(a), \delta)=M(u y(a), \delta),
$$

и значит, $M(x y(a), \delta) \subseteq M(u y(a), \delta)$. Аналогичными рассуждениями из (4) получаем, что $M(u y(a), \delta) \subseteq M(u v(a), \delta)$. Поэтому $x y(a) \in M(x y(a), \delta) \subseteq M(u v(a), \delta)$. Это означает, что $\sigma_{x y} \in M\left(\sigma_{u v}, \varphi(\delta)\right)$ согласно (1). Таким образом, справедливо включение (2). Следовательно, соответствие $\varphi: \delta \rightarrow \varphi(\delta)$ является отображением $\mathfrak{I}(\mathfrak{U})$ в $\mathfrak{I}(S(\mathfrak{U}))$. Используя равенство (1), заключаем, что

$$
\delta_{1} \subseteq \delta_{2} \Longleftrightarrow \varphi\left(\delta_{1}\right) \subseteq \varphi\left(\delta_{2}\right)
$$

для любых двух топологий $\delta_{1}, \delta_{2} \in \mathfrak{s}(\mathfrak{U})$.

Осталось показать, что отображение $\varphi$ сюръективно. Пусть $\varkappa \in \mathfrak{I}(S(\mathfrak{U}))$ и $u \in \Omega^{*}$. Положим

$$
K(u)=\left\{v(a) \in A \mid \sigma_{v} \in M\left(\sigma_{u}, \varkappa\right)\right\}
$$

Непосредственной проверкой убеждаемся, что совокупность подмножеств множества $A$ вида $K(u)$, где $u \in \Omega^{*}$, образует базу некоторой топологии $\delta$ на этом множестве и $K(u)=M(u(a), \delta)$ при всех $u \in \Omega^{*}$. Убедимся, что $\delta \in \mathfrak{I}(\mathfrak{U})$. Для этого покажем, что $f(M(u(a), \delta)) \subseteq M(f u(a), \delta)$ для любой операции $f \in \Omega$ и элемента $u(a) \in A$.

Действительно, если $v(a)=f(w(a))$, где $w(a) \in M(u(a), \delta)$, то $\sigma_{w} \in M\left(\sigma_{u}, \varkappa\right)$ ввиду (5). Поэтому $\sigma_{f w}=\sigma_{f} \sigma_{w} \in M\left(\sigma_{f}, \varkappa\right) M\left(\sigma_{u}, \varkappa\right)$. Однако, $\varkappa \in \mathfrak{s}(S(\mathfrak{U}))$, и значит, $M\left(\sigma_{f}, \varkappa\right) M\left(\sigma_{u}, \varkappa\right) \subseteq M\left(\sigma_{f u}, \varkappa\right)$ согласно лемме 1 из [2].

Следовательно, $\sigma_{f w} \in M\left(\sigma_{f u}, x\right)$, откуда $f w(a) \in M(f u(a), \delta)$ в силу (5). Применяя снова лемму 1 из [11], получаем, что $\delta \in \mathfrak{I}(\mathfrak{U})$. Осталось проверить, что $\varphi(\delta)=\varkappa$, то есть 
$M\left(\sigma_{u}, \varkappa\right)=M\left(\sigma_{u}, \varphi(\delta)\right)$ для любого элемента $\sigma_{u} \in S(\mathfrak{U})$. Действительно, из равенств (1) и (5) следует, что

$$
\sigma_{v} \in M\left(\sigma_{u}, \varphi(\delta)\right) \Longleftrightarrow v(a) \in M(u(a), \delta) \Longleftrightarrow \sigma_{v} \in M\left(\sigma_{u}, \varkappa\right)
$$

для каждого $\sigma_{v} \in S(\mathfrak{U})$. Таким образом, отображение $\varphi: \mathfrak{I}(\mathfrak{U}) \rightarrow \mathfrak{I}(S(\mathfrak{U}))-$ изоморфизм решеток.

Напомним (см. [10]), что унарная алгебра $\mathfrak{U}$ называется сильно связной, если она порождается любым своим элементом. Согласно теореме 1 из [10], характеристическая полугруппа $S(\mathfrak{U})$ коммутативной сильно связной алгебры $\mathfrak{U}$ является абелевой группой.

Теперь, используя результаты из [12], из теоремы 1 получаем следующее утверждение.

Следствие 1. Решетка топологий произвольной конечной коммутативной сильно связной алгебры модулярна.

Следующий пример показывает, что условие конечности алгебры в этом утверждении существенно.

Пример 1. Пусть $\mathfrak{C}_{Z}=\langle\mathbf{Z}, f, g\rangle$ - алгебра с двумя унарными операциями $f$ и $g$, где $f(z)=z+1, g(z)=z-1$ для всех $z \in \mathbf{Z}$. Ясно, что эта алгебра коммутативна и сильно связна. Покажем, что решетка $\mathfrak{s}\left(\mathfrak{c}_{Z}\right)$ топологий этой алгебры немодулярна.

Пусть $X$ - множество всех неположительных целых чисел, $Y$ - множество всех четных неотрицательных целых чисел, и $\varkappa=t\left(\mathbf{N}_{0}\right), \delta=t(X), v=t(Y)-$ топологии на алгебре $\mathfrak{c}_{Z}$, порожденные множествами $\mathbf{N}_{0}, X, Y$ соответственно.

Отсюда следует, что $\mathbf{N}_{0}=Y \cup g^{-1}(Y) \in v$, и значит, $x \leqslant v$. Убедимся также, что $\varkappa \neq v$. Для этого проверим, что $Y \notin \varkappa$.

Предположим, что $Y \in \varkappa$. Из определения операции $f$ в алгебре $\mathfrak{c}_{Z}$ вытекает, что

$$
f^{m}\left(\mathbf{N}_{0}\right)=\{z \mid z \in \mathbf{Z}, z \geqslant m\}
$$

для любого числа $m \in \mathbf{Z}$. Кроме того, $g^{-1}(U)=f(U)$ для любого множества $U \subseteq \mathbf{Z}$. Поэтому, в силу леммы 1 , множество $Y$ является объединением некоторых множеств вида $f^{s}\left(\mathbf{N}_{0}\right)$, где $s \in \mathbf{Z}$. Учитывая, что $0 \in Y$, получим, что включения $0 \in f^{k}\left(\mathbf{N}_{0}\right) \subseteq Y$ справедливы для некоторого $k \in \mathbf{Z}$. Это означает, что $k \leqslant 0$, откуда следует, что $1 \in f^{k}\left(\mathbf{N}_{0}\right)$, что невозможно, поскольку $1 \notin Y$.

Теперь для доказательства немодулярности решетки $\mathfrak{s}\left(\mathfrak{c}_{Z}\right)$ достаточно установить, что $\varkappa \vee(\delta \wedge v)=\varkappa$ и $(\varkappa \vee \delta) \wedge v=v$.

Проверим, что $\varkappa \vee(\delta \wedge v)=\varkappa$. Для этого заметим, что при любом $k \in \mathbf{Z}$ справедливы равенства

$$
f^{k}(X)=\{z \in \mathbf{Z} \mid z \leqslant k\}, \quad f^{k}(Y)=\{z \in \mathbf{Z} \mid z \geqslant k \& z \equiv k \quad(\bmod 2)\} .
$$

Далее, пусть $T \in \delta \wedge v$. Если допустить, что $T \neq \varnothing$ и $T \neq \mathbf{Z}$, то, используя лемму 1 , получим, что $T$ бесконечно и ограничено сверху и снизу. Это невозможно. Поэтому $\delta \wedge v=0_{\mathfrak{s}\left(\mathfrak{E}_{Z}\right)}$, и значит, $x \vee(\delta \wedge v)=\varkappa$.

Покажем, что $\varkappa \vee \delta=1_{\mathfrak{\Im}\left(\mathfrak{夭}_{Z}\right)}$. Из определения топологий $\varkappa$ и $\delta$ вытекает, что множество $\{0\}=\mathbf{N}_{0} \cap X \in \varkappa \vee \delta$. Кроме того, $\{k\}=f^{k}(\{0\})$ для любого $k \in \mathbf{Z}$. Следовательно, $\varkappa \vee \delta=1_{\mathfrak{I}\left(\mathfrak{c}_{Z}\right)}$, откуда очевидным образом вытекает равенство $(\varkappa \vee \delta) \wedge v=v$. Таким образом, решетка $\mathfrak{s}\left(\mathfrak{c}_{Z}\right)$ топологий алгебры $\mathfrak{c}_{Z}$ немодулярна. 


\section{4. Конечность линейно-упорядоченных решеток топологий коммутативных унарных алгебр}

Лемма 2. Если решетка топологий коммутативной унарной алгебры $\mathfrak{U}=\langle A, \Omega\rangle$ является ияепью, то эта алгебра сильно связна.

Доказательство. Допустим, что существует элемент $a \in A$ такой, что $A \neq(a)$. Зафиксируем также произвольный элемент $b \in A \backslash(a)$ и положим

$$
\delta_{1}=t((a)), \quad \delta_{2}=t(\{b\}) .
$$

Если предположить, что $\delta_{1} \leqslant \delta_{2}$, то $(a) \in t(\{b\})$, и значит, согласно лемме 1 , множество (a) равно объединению некоторых конечных пересечений множеств вида $u^{-1}(\{b\})$, где $u \in \Omega^{*}$. Поэтому $a \in u_{0}^{-1}(\{b\})$ для некоторого $u_{0} \in \Omega^{*}$, откуда $b=u_{0}(a) \in(a)$, что противоречит выбору элемента $b$.

Пусть теперь $\delta_{2} \leqslant \delta_{1}$. Тогда $\{b\} \in t((a))$. Отсюда в силу леммы 1 вытекает, что

$$
\{b\}=u_{1}^{-1}((a)) \cap u_{2}^{-1}((a)) \cap \ldots \cap u_{k}^{-1}((a)),
$$

где $k \in \mathbf{N}, u_{1}, u_{2}, \ldots, u_{k} \in \Omega^{*}$. Кроме того, $a \in u_{1}^{-1}((a)) \cap u_{2}^{-1}((a)) \cap \ldots \cap u_{k}^{-1}((a))$, поскольку $u_{1}(a), u_{2}(a), \ldots, u_{k}(a) \in(a)$. Следовательно, $a=b$, что снова противоречит выбору элемента $b$.

Таким образом, элементы $\delta_{1}$ и $\delta_{2}$ решетки $\mathfrak{I}(\mathfrak{U})$ не сравнимы между собой, что противоречит условию.

Напомним (см. лемму 2 в [4]), что классы каждой конгруэнции $\theta$ алгебры $\mathfrak{U}$ образуют базу некоторой топологии $\tau(\theta)$, которую мы будем называть топологией, индуцированной конгруэнцией $\theta$.

Лемма 3. Пусть $\mathfrak{U}=\langle A, \Omega\rangle-$ сильно связная коммутативная унарная алгебра такая, что $S(\mathfrak{U}) \cong \mathbf{Z}_{p} \infty$ для некоторого простого числа $p$ (через $\mathbf{Z}_{p} \infty$, как обычно, обозначается группа типа $p^{\infty}$ ). Тогда решетка топологий этой алгебры не является цеепью.

Доказательство. Зафиксируем элемент $a \in A$. По условию, для любых $x, y \in A$ найдутся $u, v \in \Omega^{*}$ такие, что $x=u(a), y=v(a)$. Положим по определению

$$
x \theta y \Longleftrightarrow \sigma_{u}^{p}=\sigma_{v}^{p} .
$$

Это определение корректно, так как из равенства $w(a)=z(a)$ всякий раз следует, что $\sigma_{w}=\sigma_{z}$. Непосредственная проверка показывает, что $\theta \in \operatorname{Con} \mathfrak{A}$.

Убедимся, что элементы $\delta_{1}=\tau(\theta)$ и $\delta_{2}=t(A \backslash\{a\})$ решетки $\mathfrak{I}(\mathfrak{U})$ не сравнимы. Для этого покажем сначала, что $A \backslash\{a\} \notin \delta_{1}$. Пусть $\sigma_{u}-$ произвольный элемент группы $S(\mathfrak{U})$, порядок которого равен $p$. Тогда $u(a) \in A \backslash\{a\}$ и $(a, u(a)) \in \theta$ в силу (6). Следовательно, $A \backslash\{a\} \notin \tau(\theta)=\delta_{1}$ по определению топологии, индуцированной конгруэнцией. Это означает, что включение $\delta_{2} \subseteq \delta_{1}$ невозможно.

Если предположить теперь, что $\delta_{1} \subseteq \delta_{2}$, то $[a]_{\theta} \in \delta_{2}$, где $[a]_{\theta}-$ класс конгруэнции $\theta$, содержащий элемент $a \in \mathfrak{U}$. Отсюда, ввиду леммы 2, вытекает, что

$$
A \backslash\left\{u_{1}(a), u_{2}(a), \ldots, u_{k}(a)\right\}=u_{1}(A \backslash\{a\}) \cap u_{2}(A \backslash\{a\}) \cap \ldots \cap u_{k}(A \backslash\{a\}) \subseteq[a]_{\theta}
$$

для некоторых $k \in \mathbf{N}, u_{1}, u_{2}, \ldots, u_{k} \in \Omega^{*}$, и значит, множество $[a]_{\theta}$ бесконечно. 
С другой стороны, если элемент $u(a) \in[a]_{\theta}$, то из (6) получаем, что $\sigma_{u}^{p}=\sigma_{e}^{p}$, где $\sigma_{e}(a)=e(a)=a$ (то есть $\sigma_{e}-$ единица группы $\left.S(\mathfrak{Q})\right)$. Следовательно, $\sigma_{u}^{p}=\sigma_{e}$. Поскольку $S(\mathfrak{V}) \cong \mathbf{Z}_{p} \infty$, в группе $S(\mathfrak{U})$ содержится лишь $p$ элементов, порядок которых равен 1 или $p$. Кроме того, как уже отмечалось, для любых $w, z \in \Omega^{*}$ справедлива эквиваленция

$$
\sigma_{w}=\sigma_{z} \Longleftrightarrow w(a)=z(a) .
$$

Таким образом, $\left|[a]_{\theta}\right|=p$, что противоречит бесконечности множества $[a]_{\theta}$.

Однопорожденный унар $\langle A, f\rangle$ (то есть алгебра с одной унарной операцией) с порождающим элементом $a$ и определяющим соотношением $a=f^{n}(a)$, где $n \in \mathbf{N}$, обозначается через $C_{n}^{0}$ и называется циклом длины $n$.

Теорема 2. Решетка топологий произвольной коммутативной унарной алгебры $\mathfrak{U}=\langle A, \Omega\rangle$ есть иепь тогда и только тогда, когда для некоторой операиии $f \in \Omega$ редукт $\mathfrak{A}_{f}=\langle A, f\rangle$ является ииклом длины $p^{k}$, где $p-$ простое число, $k \in \mathbf{N}_{0}$.

Доказательство. Докажем достаточность. Из определений вытекает, что решетка $\mathfrak{I}(\mathfrak{V})$ топологий унарной алгебры $\mathfrak{A}$ является подрешеткой решетки топологий каждого ее редукта $\mathfrak{A}_{f}, f \in \Omega$. Следовательно, если $\mathfrak{\mathfrak { H }}_{f} \cong C_{p^{k}}^{0}$, где $f \in \Omega, p$ - простое число и $k \in \mathbf{N}_{0}$, то $\mathfrak{I}(\mathfrak{U})$ - цепь согласно теореме 7 из [4].

Докажем необходимость. Пусть решетка топологий коммутативной унарной алгебры $\mathfrak{U}$ - цепь. Тогда, в силу теоремы 1 из [4], решетка Con $\mathscr{U}$ конгруэнций этой алгебры также является цепью. Кроме того, $\mathfrak{A}$ - сильно связная алгебра в силу леммы 2. Отсюда, по теореме 1 из [1], характеристическая полугруппа $S(\mathfrak{U})$ является абелевой группой и $\operatorname{Con} \mathfrak{A} \cong \operatorname{Sub} S(\mathfrak{U})$. Теперь из теоремы Оре (см., напр., [5], с. 276) следует, что группа $S(\mathfrak{U})$ изоморфна либо группе типа $p^{\infty}$, либо циклической группе $\mathbf{Z}_{p^{k}}$ порядка $p^{k}$, где $k \in \mathbf{N}_{0}$ и $p-$ простое число.

Учитывая лемму 3 , остается принять, что $S(\mathfrak{V}) \cong \mathbf{Z}_{p^{k}}$. Наконец, из определения группы $S(\mathfrak{U})$ вытекает, что совокупность элементов вида $\sigma_{f}$, где $f \in \Omega$, является системой порождающих этой группы. Поэтому найдется операция $f \in \Omega$, для которой порядок элемента $\sigma_{f}$ равен $p^{k}$. Это, в свою очередь, означает, что $\mathfrak{U}_{f} \cong C_{p^{k}}^{0}$.

Из теоремы 2 непосредственно вытекают следующие утверждения.

Следствие 2. Решетка топологий коммутативной унарной алгебры $\mathfrak{Q}$ является цепью тогда и только тогда, когда $\mathfrak{Q}$ - сильно связная алгебра и Aut $\mathfrak{Q} \cong \mathbf{Z}_{p^{k}}$, где $p$ - простое число, $k \in \mathbf{N}_{0}$.

Следствие 3. Произвольная цеепь изоморфна решетке топологий подходящей коммутативной унарной алгебры в том и только том случае, когда она конечна.

\section{5. Об условиях конечности решеток топологий и конгруэнций коммутативных унарных алгебр}

Элементы $a$ и $b$ унарной алгебры $\mathscr{U}$ называются взаимно достижимыми (см. с. 66 в [15]), если $(a)=(b)$. Очевидно, что отношение взаимной достижимости является эквивалентностью. Класс этой эквивалентности с порождающим элементом $a$ называется слоем этого элемента и обозначается через $L(a)$. 
Лемма 4. Любой неодноэлементный слой произвольной коммутативной унарной алгебры $\mathfrak{U}=\langle A, \Omega\rangle$ является подалгеброй некоторого ее редукта $\left\langle A, \Omega_{1}\right\rangle$, где $\Omega_{1} \neq \varnothing$.

Доказательство. Пусть $|L(a)|>1$ для некоторого элемента $a \in A$. Тогда множество

$$
\Omega_{a}=\left\{u \in \Omega^{*} \mid u(a) \in L(a)\right\}
$$

содержит хотя бы одно непустое слово. Обозначим через $\Omega_{1}$ множество всех сигнатурных символов, каждый из которых входит хотя бы в одно слово из $\Omega_{a}$. Покажем, что $\left\langle L(a), \Omega_{1}\right\rangle$ - сильно связная подалгебра редукта $\mathfrak{U}_{1}=\left\langle A, \Omega_{1}\right\rangle$ алгебры $\mathfrak{U}$.

Для этого убедимся сначала, что $L(a)-$ подалгебра редукта $\mathfrak{U}_{1}=\left\langle A, \Omega_{1}\right\rangle$. Пусть $f \in \Omega_{1}$ и $b \in L(a)$. Тогда в силу коммутативности алгебры $\mathfrak{U}$ найдется слово $u \in \Omega^{*}$, для которого $u f(a) \in L(a)$, откуда $(a)=(u f(a))$. Кроме того, нетрудно проверить, что элементы $f(a)$ и $f(b)$ взаимно достижимы.

Следовательно,

$$
(a)=(u f(a)) \subseteq(f(a))=(f(b)) \subseteq(b)=(a),
$$

откуда $(f(b))=(a)$, то есть $f(b) \in L(a)$.

Вторая часть предложения вытекает из того факта, что $(a)=(b)$ для любых элементов $a, b \in L(a)$, и определения множества $\Omega_{1}$.

В [16] показано, что условие конечности решетки конгруэнций произвольного унара равносильно конечности самого унара. В дальнейшем в [13] было установлено, что это утверждение справедливо и для решеток топологий унаров. В настоящей работе эти результаты обобщены для произвольных коммутативных унарных алгебр.

Теорема 3. Решетка конгруэнций коммутативной унарной алгебры $\mathfrak{U}=\langle A, \Omega\rangle$ конечна тогда и только тогда, когда сама алгебра $\mathfrak{U}$ конечна.

Доказательство. Предположим, что алгебра $\mathfrak{U}$ бесконечна, но решетка Con $\mathfrak{U}$ конечна. Тогда конечной будет и решетка $\mathrm{Sub} \mathfrak{A}$, поскольку она вложима в Con $\mathfrak{A}$. Кроме того, очевидно, что если $L(x) \neq L(y)$, то $(x) \neq(y)$. Поэтому алгебра $\mathfrak{U}$ имеет лишь конечное число слоев. Отсюда в силу бесконечности алгебры $\mathfrak{U}$ найдется хотя бы один бесконечный слой $L(a), a \in A$. На множестве $(a)$ определим отношение $\rho$, положив

$$
x \rho y \Longleftrightarrow(x=y) \vee(\{x, y\} \cap L(a)=\varnothing)
$$

для любых $x, y \in(a)$.

Очевидно, что $\rho$ - эквивалентность. Проверим, что $\rho-$ конгруэнция алгебры $(a)$.

Пусть $f \in \Omega, x, y \in(a), x \rho y$ и $x \neq y$. Предположим, что $f(x) \in L(a)$. Тогда $(a)=(f(x)) \subseteq(x)$, откуда следует, что $(a)=(x)$, поскольку $x \in(a)$. Следовательно, $x \in L(a)$ в силу (7), что противоречит неравенству $x \neq y$. Это означает, что $f(x) \notin L(a)$. Аналогично получаем, что $f(y) \notin L(a)$. Таким образом, $f(x) \rho f(y)$.

Покажем, что решетка конгруэнций фактор-алгебры $\mathfrak{B}=(a) / \rho$ бесконечна. По лемме 4 , слой $L(a)$ - сильно связная подалгебра некоторого редукта алгебры $\left\langle(a), \Omega_{1}\right\rangle$, где $\Omega_{1} \neq \varnothing$. Убедимся, что решетка конгруэнций алгебры $\mathfrak{L}=\left\langle L(a), \Omega_{1}\right\rangle$ бесконечна.

По теореме 1 из [1], характеристическая полугруппа $S(\mathfrak{R})$ этой алгебры является бесконечной абелевой группой и $\operatorname{Con} \mathfrak{R} \cong \operatorname{Sub} S(\mathfrak{L})$. Это означает, что решетка Con $\mathfrak{Z}$ бесконечна. 
Далее, для любой конгруэнции $\theta \in \mathrm{Con} \mathfrak{R}$ зададим бинарное отношение $\varphi(\theta)$ на носителе алгебры $\mathfrak{B}$ по правилу:

$$
[c]_{\rho} \varphi(\theta)[d]_{\rho} \Longleftrightarrow(c \rho d) \vee(\{c, d\} \subseteq L(a) \&(c \theta d)
$$

для любых $c, d \in(a)$.

Непосредственной проверкой убеждаемся, что это определение корректно и $\varphi(\theta)-$ эквивалентность. Покажем, что это отношение стабильно относительно любой операции $f \in \Omega$. Пусть $\left([x]_{\rho},[y]_{\rho}\right) \in \varphi(\theta)$. Если при этом $x \rho y$, то $f(x) \rho f(y)$, и значит, $\left([f(x)]_{\rho},[f(y)]_{\rho}\right) \in \varphi(\theta)$ согласно $(8)$.

Пусть $\{x, y\} \subseteq L(a)$ и $x \theta y$. Тогда $(x)=(a)=(y)$, откуда в силу условия коммутативности алгебры вытекает, что $(f(x))=(f(y))$. По определению слоя возможны следующие случаи:

(1) $\{f(x), f(y)\} \subseteq L(a)$,

(2) $\{f(x), f(y)\} \cap L(a)=\varnothing$.

В первом случае $f(x) \theta f(y)$, поскольку $x \quad \theta y$. Отсюда в силу (8) следует, что $\left([f(x)]_{\rho},[f(y)]_{\rho}\right) \in \varphi(\theta)$.

Во втором случае из (7) следует, что $f(x) \rho f(y)$, откуда в силу (8) получаем, что $\left([f(x)]_{\rho},[f(y)]_{\rho}\right) \in \varphi(\theta)$.

Таким образом, соответствие $\theta \rightarrow \varphi(\theta)$ является отображением решетки Con $\mathfrak{R}$ решетку Con $\mathfrak{B}$. В силу (8) оно инъективно. Следовательно, решетка $\operatorname{Con} \mathfrak{B}$ бесконечна, поскольку Con $\mathfrak{\imath}$ бесконечна.

Далее, алгебра $\mathfrak{B}$ - фактор-алгебра алгебры $(a)$, поэтому $|\operatorname{Con} \mathfrak{B}| \leqslant|\operatorname{Con}(a)|$ (см., напр., с. 61 в [17]). Кроме того, $|\operatorname{Con}(a)| \leqslant|\operatorname{Con} \mathfrak{U}|$ в силу леммы 1 в [18]. Следовательно, решетка Con $\mathfrak{U}$ бесконечна.

Из теоремы 3 и теоремы 1 из [4] вытекает следующее утверждение.

Следствие 4. Решетка $\mathfrak{I}(\mathfrak{U})$ топологий произвольной коммутативной унарной алгебры конечна тогда и только тогда, когда алгебра $\mathfrak{A}$ конечна.

Покажем теперь, что если бесконечная алгебра $\mathfrak{U}$ не является унарной или не коммутативна, то каждая из решеток $\mathfrak{I}(\mathfrak{U})$ и Con $\mathfrak{U}$ может быть конечной. С этой целью докажем сначала следующее вспомогательное утверждение.

Лемма 5. Пусть $\mathfrak{C}=\langle C, \cdot\rangle-$ произвольный группоид и $\Omega-$ функциональная сигнатура, содержащая хотя бы один символ, арность которого больше 1. Тогда на множестве $C$ можно построить алгебру $\mathfrak{U}$ сигнатуры $\Omega$ такую, что $\mathfrak{I}(\mathfrak{C}) \cong \mathfrak{I}(\mathfrak{U})$.

Доказательство. Интерпретируем на множестве $C$ символы из $\Omega$ следующим образом. Пусть $F \in \Omega$ и арность символа $F$ равна $m$. Положим $F(x)=x$ для любого $x \in C$ при $m=1$ и $F\left(x_{1}, x_{2}, \ldots, x_{m}\right)=x_{1} \cdot x_{2}$ для любых $x_{1}, x_{2}, \ldots, x_{m} \in C$ при $m>1$, где $\cdot-$ операция группоида $\mathfrak{C}$. Непосредственная проверка показывает, что каждая топология на группоиде $\mathfrak{c}$ будет топологией на алгебре $\mathfrak{U}=\langle C, \Omega\rangle$ и, обратно, всякая топология на алгебре $\mathfrak{U}$ является топологией на группоиде $\mathfrak{c}$.

Теорема 4. Пусть $\Omega$ - произвольная функииональная сигнатура, содержашая хотя бы один символ, арность которого больше 1. Тогда для любого ичелого числа $n \geqslant 2$ существует бесконечная алгебра $\mathfrak{U}$ сигнатуры $\Omega$, решетка топологий которой содержит ровно $n$ элементов. 
Доказательство. Согласно лемме 5, достаточно построить для любого целого числа $n \geqslant 2$ бесконечный группоид с $n$-элементной решеткой топологий. Зафиксируем произвольное целое число $n \geqslant 2$ и определим бинарную операцию $*$ на множестве $\mathbf{N}$ по правилу

$$
x * y= \begin{cases}\min \{x, y\}, & \text { если } y=x+1 \text { или } \min \{x, y\} \leqslant n-2, x \neq y, \\ x+1 & \text { в остальных случаях. }\end{cases}
$$

Докажем, что решетка $\mathfrak{s}(\mathfrak{C})$ группоида $\mathfrak{V}_{n}=\langle\mathbf{N}, *\rangle$ содержит ровно $n$ элементов.

Зафиксируем произвольное число $i \in\{1,2, \ldots, n-2\}$. Очевидно, что совокупность подмножеств $\{1\},\{2\}, \ldots,\{i\}, \mathbf{N} \backslash\{1,2, \ldots, i\}$ является базой некоторой топологии $\delta_{i}$ на множестве N. Проверим, что $\delta_{i} \in \mathfrak{s}\left(\mathfrak{C}_{n}\right)$. Действительно, пусть $x * y=z$ для некоторых $x, y, z \in \mathbf{N}$ и $U_{z}-$ произвольная окрестность элемента $z$ в топологии $\delta_{i}$. Убедимся, что найдутся окрестности $U_{x}$ и $U_{y}$ в топологии $\delta_{i}$ элементов $x$ и $y$, соответственно, такие, что $U_{x} * U_{y} \subseteq U_{z}$. Рассмотрим возможные случаи.

Если $x \leqslant i$ и $y \leqslant i$, то $\{x\},\{y\} \in \delta_{i}$. В этом случае достаточно положить $U_{x}=\{x\}$ и $U_{y}=\{y\}$.

В случае $x>i, y \leqslant i$ справедливы равенства $z=x * y=y$ по определению операции *. С другой стороны, $\{y\}, \mathbf{N} \backslash\{1, \ldots, i\} \in \delta_{i}$. Полагая $U_{x}=\mathbf{N} \backslash\{1,2, \ldots, i\}$ и $U_{y}=\{y\}$, получаем, что $U_{x} * U_{y}=\{y\} \subseteq U_{z}$. Случай $x \leqslant i$ и $y>i$ рассматривается аналогично.

Наконец, пусть $x>i, y>i$. Тогда, поскольку $z \geqslant \min \{x, y\}$, справедливо неравенство $z>i$, и значит, $\mathbf{N} \backslash\{1,2, \ldots, i\} \subseteq U_{z}$ по определению топологии $\delta_{i}$. Используя включение $N \backslash\{1,2, \ldots, i\} * \mathbf{N} \backslash\{1,2, \ldots, i\} \subseteq \mathbf{N} \backslash\{1,2, \ldots, i\}$, можно положить

$$
U_{x}=U_{y}=\mathbf{N} \backslash\{1,2, \ldots, i\} .
$$

Следовательно, $\delta_{i} \in \mathfrak{I}\left(\mathfrak{G}_{n}\right)$.

Далее через $\delta_{0}$ и $\delta_{n-1}$ обозначим, соответственно, антидискретную и дискретную топологию на группоиде $\mathfrak{c}_{n}$. Очевидно, что $\delta_{i} \neq \delta_{j}$ при $i, j \in\{0,1, \ldots, n-1\}, i \neq j$. Пусть теперь $\delta \in \mathfrak{I}\left(\mathfrak{C}_{n}\right)$. Проверим, что $\delta \in\left\{\delta_{0}, \delta_{1}, \ldots, \delta_{n-1}\right\}$.

Для этого убедимся сначала в справедливости импликации

$$
\{k\} \in \delta \Longrightarrow\{k-1\} \in \delta
$$

для любого целого числа $k>1$.

Действительно, ввиду равенства $(k-1) *(k-1)=k$ найдутся окрестности $U_{k-1}$ и $U_{k-1}^{\prime}$ элемента $k-1$ такие, что $U_{k-1} * U_{k-1}^{\prime}=\{k\}$. Отсюда следует равенство $U_{k-1}=\{k-1\}$, поскольку $m *(k-1) \neq k$ при всех $m \neq k-1$. Это означает, что $\{k-1\} \in \delta$.

Предположим теперь, что $\{n-1\} \in \delta$. Покажем, что в этом случае $\delta-$ дискретная топология. В силу (9) получаем, что $\{n-2\} \in \delta,\{n-3\} \in \delta, \ldots,\{1\} \in \delta$.

Пусть $\{k\} \in \delta$ для некоторого целого числа $k \geqslant n-1$. Покажем, что $\{k+1\} \in \delta$. Действительно, $k *(k+1)=k$, откуда $U_{k} * U_{k+1}=\{k\}$ для некоторых окрестностей $U_{k}$ и $U_{k+1}$ в топологии $\delta$ элементов $k$ и $k+1$, соответственно. Поэтому $\{k+1\}=U_{k+1} \in \delta$, так как $k * m \neq k$ при всех $m \neq k+1$. Таким образом, при выполнении соотношения $\{n-1\} \in \delta$ справедливо включение $\{m\} \in \delta$ при любом $m \in \mathbf{N}$, и значит, $\delta=\delta_{n-1}$.

Пусть $\{n-1\} \notin \delta$. Докажем, что в этом случае $\delta=\delta_{j}$ для некоторого числа $j \in\{0,1, \ldots, n-2\}$. Для каждого элемента $k \in \mathbf{N} \backslash\{1,2, \ldots, n-2\}$ через $O_{k}$ обозначим пересечение всех окрестностей элемента $k$ и докажем включение

$$
\mathbf{N} \backslash\{1,2, \ldots, n-2\} \subseteq O_{k} .
$$


Покажем сначала, что (10) справедливо при $k=n-1$. Доказательство будем вести индукцией по числу $l \in \mathbf{N} \backslash\{1,2, \ldots, n-2\}$, начиная с $l=n-1$. Тогда очевидно, что $n-1 \in O_{n-1}$. Предположим теперь, что $l \in O_{n-1}$ для некоторого $l \geqslant n-1$. Нужно показать, что $l+1 \in O_{n-1}$. С этой целью зафиксируем произвольную окрестность $U$ элемента $n-1$ и убедимся, что $l+1 \in U$.

В силу равенства $(n-1) * n=n-1$ найдутся окрестности $U_{n-1}$ и $U_{n}$ элементов $n-1$ и $n$, соответственно, такие, что $U_{n-1} * U_{n} \subseteq U$. Если $l \neq n-1$, то $l * n=l+1$, откуда $l+1 \in U_{n-1} * U_{n} \subseteq U$, поскольку по индуктивному предположению $l \in U_{n-1}$.

Пусть $l=n-1$. Покажем сначала, что $U_{n} \backslash\{1,2, \ldots, n-2, n\} \neq \varnothing$. Действительно, допустим, что

$$
U_{n} \subseteq\{1,2, \ldots, n-2\} \cup\{n\}
$$

Убедимся в том, что $\{1,2, \ldots, n-2\} \cap O_{n} \neq \varnothing$. Если предположить, что $1 \notin W_{n}^{1}$, $2 \notin W_{n}^{2}, \ldots, n-2 \notin W_{n}^{n-2}$ для некоторых окрестностей $W_{n}^{1}, W_{n}^{2}, \ldots, W_{n}^{n-2}$ элемента $n$, то согласно (11) получаем, что $\{n\}=U_{n} \cap W_{n}^{1} \cap W_{n}^{2} \cap \ldots \cap W_{n}^{n-2} \in \delta$, откуда $\{n-1\} \in \delta$ в силу (9), что невозможно.

Пусть $l$ - наибольшее число в множестве $\{1,2, \ldots, n-2\} \cap O_{n}$ и $V_{n}-$ произвольная окрестность элемента $n$. Тогда $l \in V_{n}$ и ввиду равенства $l * n=l$ найдется окрестность $V_{n}^{\prime}$ элемента $n$ такая, что $\{l\} * V_{n}^{\prime} \subseteq V_{n}$, откуда следует, что $l+1=l * l \in V_{n}$. Отсюда вытекает, что $l+1 \in O_{n}$, и значит, $l+1 \notin\{1,2, \ldots, n-2\}$ в силу выбора элемента $l$. Следовательно, $n-1=l+1 \in O_{n} \subseteq U_{n}$, что противоречит (11). Поэтому $U_{n} \backslash\{1,2, \ldots, n-2, n\} \neq \varnothing$. Зафиксируем произвольный элемент $z \in U_{n} \backslash\{1,2, \ldots, n-2, n\}$. Снова используя индуктивное предположение и включение $U_{n-1} * U_{n} \subseteq U$, получаем, что $l+1=l * z \in U$, откуда $l+1 \in O_{n-1}$.

Таким образом, при $k=n-1$ включение (10) справедливо. Теперь проведем индукцию по числу $k$. Предположим, что (10) справедливо при некотором $k \geqslant n-1$. Докажем, что $\mathbf{N} \backslash\{1,2, \ldots, n-2\} \subseteq O_{k+1}$. Пусть $V \in \delta$ и $k+1 \in V$. Тогда в силу равенства $k * k=k+1$ найдутся окрестности $V_{k}$ и $V_{k}^{\prime}$ элемента $k$, для которых $V_{k} * V_{k}^{\prime} \subseteq V$. Так как $\mathbf{N} \backslash\{1,2, \ldots, n-2\} \subseteq \mathbf{N} \backslash\{1,2, \ldots, n-2\} * \mathbf{N} \backslash\{1,2, \ldots, n-2\}$, в силу индуктивного предположения $\mathbf{N} \backslash\{1,2, \ldots, n-2\} \subseteq V_{k} * V_{k}^{\prime} \subseteq V$. Поэтому $\mathbf{N} \backslash\{1,2, \ldots, n-2\} \subseteq O_{k+1}$. Таким образом, включение (10) справедливо для любого числа $k \in \mathbf{N} \backslash\{1,2, \ldots, n-2\}$.

Далее положим

$$
\mathfrak{M}=\{X \mid X \in \delta \& \mathbf{N} \backslash\{1,2, \ldots, n-2\} \subseteq X\}, \quad M=\bigcap_{X \in \mathfrak{M}} X
$$

Очевидно, что множество $\mathfrak{M}$ конечно, откуда $M \in \delta$. Убедимся теперь, что

$$
k \in U \cap M \Longrightarrow k+1 \in U \cap M
$$

для любых $U \in \delta$ и $k \in\{1,2, \ldots, n-2\}$.

Пусть $k \in U \cap M$, где $U \in \delta$ и $k \in\{1,2, \ldots, n-2\}$. Тогда $U \cap M \in \delta$, откуда ввиду равенства $k *(n-1)=k$ следует, что $U_{k} * U_{n-1} \subseteq U \cap M$ для некоторых окрестностей $U_{k}, U_{n-1} \in \delta$ элементов $k$ и $n-1$, соответственно. В силу (10) получаем, что $U_{n-1} \in \mathfrak{M}$, и значит, $M \subseteq U_{n-1}$ согласно (12). Поэтому $k \in M \subseteq U_{n-1}$.

Следовательно,

$$
k+1=k * k \in U_{k} * U_{n-1} \subseteq U \cap M .
$$


Таким образом, соотношение (13) справедливо для любого $U \in \delta$ и любого числа $k \in\{1,2, \ldots, n-2\}$. Пусть $j-$ наименьшее число в множестве $M$ и $i=j-1$. Из (12) вытекает, что $i \in\{0,1, \ldots, n-2\}$. Покажем, что $\delta=\delta_{i}$. Для этого проверим, что

$$
M=\mathbf{N} \backslash\{1,2, \ldots, i\} .
$$

Действительно, если $l \in M$, то $l \in \mathbf{N} \backslash\{1,2, \ldots, i\}$ в силу выбора числа $i$, и значит, $M \subseteq \mathbf{N} \backslash\{1,2, \ldots, i\}$. Докажем обратное включение. Пусть $l \in \mathbf{N} \backslash\{1,2, \ldots, i\}$. Рассмотрим возможные случаи.

Если $l>n-2$, то $l \in M$ ввиду (12). Если $l=i+1$, то $l \in M$ в силу выбора числа $i$. Наконец, пусть $i+1<l \leqslant n-2$. Тогда, последовательно применяя импликацию (13), получаем, что $l \in M$. Таким образом, $M=\mathbf{N} \backslash\{1,2, \ldots, i\}$.

Проверим, что $\{x\} \in \delta$ для любого $x \in\{1,2, \ldots, i\}$. Поскольку $i * i=i+1$ и в силу (14) множество $M$ является окрестностью элемента $i+1$, существуют окрестности $U_{i}$ и $U_{i}^{\prime}$ элемента $i$ такие, что $U_{i} * U_{i}^{\prime} \subseteq M$. Если допустить, что множество $U_{i}$ содержит некоторый элемент $m \neq i$, то $m * i \leqslant i$. Это противоречит равенству (14), так как $m * i \in M$. Следовательно, $\{i\}=U_{i} \in \delta$. Теперь из (9) вытекает, что $\{x\} \in \delta$ для любого целого числа $x \in\{1,2, \ldots, i\}$. Отсюда, используя (14), заключаем, что $\delta_{i} \subseteq \delta$ по определению топологии $\delta_{i}$.

Зафиксируем множество $U \in \delta$. Если при этом $U \cap M=\varnothing$, то $U \subseteq\{1,2, \ldots, i\}$ ввиду (14), и значит, $U \in \delta_{i}$. Пусть теперь $U \cap M \neq \varnothing$. Тогда $n-1 \in U \cap M$ в силу (13), откуда согласно (10) получаем, что

$$
\mathbf{N} \backslash\{1,2, \ldots, n-2\} \subseteq O_{n-1} \subseteq U .
$$

Поэтому $M \subseteq U$ ввиду (12). Это означает, что $U \in \delta_{i}$ по определению топологии $\delta_{i}$. Следовательно, $\delta=\delta_{i}$. Таким образом, $\left|\mathfrak{s}\left(\mathfrak{C}_{n}\right)\right|=n$.

Далее заметим, что топологии $\delta_{0}, \delta_{1}, \ldots, \delta_{n-1}$, построенные в доказательстве теоремы 4, попарно сравнимы. Отсюда вытекает следующее утверждение.

Следствие 5. Пусть $\Omega$ - произвольная функииональная сигнатура, содержащая хотя бы один символ, арность которого больше 1. Тогда для любого челого числа $n \geqslant 2$ существует бесконечная алгебра $\mathfrak{U}$ сигнатуры $\Omega$, решетка топологий которой является n-элементной ияепью.

Теорема 5. Пусть $\Omega$ - произвольная функциональная сигнатура, содержащая не менее трех унарных символов. Тогда существует бесконечная алгебра $\mathfrak{A}$ сигнатурь $\Omega$, решетка топологий которой двухэлементна, то есть состоит только из дискретной $u$ антидискретной топологий.

Доказательство. Зафиксируем три унарных символа $f, g, h \in \Omega$. Для каждого $x \in \mathbf{Z}$ положим $f(x)=x+1, g(x)=x-1$ и

$$
h(x)= \begin{cases}0, & \text { если } x=0 \\ 1, & \text { если } x \neq 0 .\end{cases}
$$

Если $F \in \Omega \backslash\{f, g, h\}$ и $m$ - арность символа $F$, то для любых $x_{1}, x_{2}, \ldots, x_{m} \in \mathbf{Z}$ положим $F\left(x_{1}, x_{2}, \ldots, x_{m}\right)=x_{1}$. Докажем, что решетка $\mathfrak{s}(\mathfrak{U})$ алгебры $\mathfrak{U}=\langle\mathbf{Z}, \Omega\rangle$ двухэлементна. Пусть $\delta \in \mathfrak{I}(\mathfrak{U})$. Если $\{0\} \in \delta$, то топология $\delta$ дискретна, поскольку $\{k\}=g^{-k}(\{0\}) \in \delta$ для любого положительного $k \in \mathbf{Z}$ и $\{k\}=f^{-k}(\{0\}) \in \delta$ для любого отрицательного $k \in \mathbf{Z}$. 
Предположим, что $\{0\} \notin \delta$. Проверим, что в этом случае топология $\delta$ антидискретна. Для этого покажем сначала, что

$$
0 \in U \Longleftrightarrow 1 \in U
$$

для любого $U \in \delta$.

Действительно, если $0 \in U$, то используя равенство $h(0)=0$, получаем, что $h\left(U_{0}\right) \subseteq U$, где $U_{0}-$ подходящая окрестность точки 0 в топологии $\delta$. Кроме того, $U_{0} \neq\{0\}$, так как $\{0\} \notin \delta$. Поэтому $1 \in U$ в силу определения функции $h$.

Пусть теперь $1 \in U$. Тогда в силу равенства $h(-1)=1$ найдется окрестность $U_{-1}$ элемента -1 такая, что $h\left(U_{-1}\right) \subseteq U$. С другой стороны, $0 \in g^{-1}\left(U_{-1}\right)$, так как $g(0)=-1 \in U_{-1}$, откуда по предыдущему $1 \in g^{-1}\left(U_{-1}\right)$, и значит, $0=g(1) \in U_{-1}$. Следовательно, $0 \in h\left(U_{-1}\right) \subseteq U$.

Покажем теперь, что

$$
0 \in U \Longrightarrow U=\mathbf{Z}
$$

для любого $U \in \delta$.

Действительно, предположим, что существует такое множество $U \in \delta$, что $0 \in U$ и $U \neq \mathbf{Z}$. Пусть $\mathbf{N} \backslash U \neq \varnothing$ и $n-$ наименьшее число в множестве $\mathbf{N} \backslash U$. Из соотношения $f^{n-1}(0)=n-1 \in U$ следует, что $0 \in f^{-(n-1)}(U)$, откуда $1 \in f^{-(n-1)}(U)$ в силу (15). Поэтому $n=f^{n-1}(1) \in U$, что противоречит выбору числа $n$.

Если $\mathbf{N} \backslash U=\varnothing$, то $\overline{\mathbf{N}} \backslash U \neq \varnothing$, где

$$
\overline{\mathbf{N}}=\{-k \mid k \in \mathbf{N}\} .
$$

Обозначим через $m$ наименьшее натуральное число такое, что $-m \in \overline{\mathbf{N}} \backslash U$. Тогда

$$
g^{m}(1)=1-m=-(m-1) \in U,
$$

то есть $1 \in g^{-m}(U)$. Поэтому $0 \in g^{-m}(U)$ в силу (15), и значит, $-m \in U$, что противоречит выбору числа $m$.

Пусть теперь $X \in \delta, X \neq \varnothing$ и $x \in X$. Тогда $0 \in f^{-x}(X)$ при $x \geqslant 0$ и $0 \in g^{-x}(X)$ при $x<0$. Отсюда в силу (16) либо $f^{-x}(X)=\mathbf{Z}$, либо $g^{-x}(X)=\mathbf{Z}$. Это означает, что $X=\mathbf{Z}$. Таким образом, $\delta=\{\varnothing, \mathbf{Z}\}$. Следовательно,

$$
|\mathfrak{s}(\mathfrak{U})|=2 .
$$

\section{Список литературы}

1. Мальцев А. И., Алгебраические системы. Наука, Москва, 1970.

2. Kilp M., Knauer U., Michalev A. V., Skornjakov L. A., Acts over monoids. A bibliographical survey of publications 1975-1981. Bibliotheks und Informationssystem der Universität Oldenburg, Oldenburg, 1982.

3. Skornjakov L. A., Unars. Colloq. Math. Soc. J. Bolyai (1982) 29, 735-743.

4. Kilp M., Knauer U., Mikhalev A. V., Acts and categories. Walter de Gruyter, Berlin, 2000.

5. Смирнов Д. М., О соответствии между регулярно определимыми многообразиями унарных алгебр и полугруппами. Алгебра и логика (1978) 17, №4, 468-477.

6. Кожухов И. Б., Полугруппы, над которыми все полигоны резидуально конечны. Фунд. прикл. матем. (1988) 4, №4, 1335-1344. 
7. Понтрягин Л. С., Непрерывные группы. Наука, Москва, 1973.

8. Arnautov V. I., Glavatsky S. T., Mikhalev A. V., Introduction to topological rings and modules. Marcel Dekker, New York, 1996.

9. Карташова А. В., Бесконечные алгебры с конечной решеткой топологий. В сб.: Тез. докл. Укр. матем. конгресса. Секция алгебры и теории чисел, 27, Киев, 2001.

10. Ésik Z., Imreh B., Remarks on finite commutative automata. Acta Cybern. (1981) 5, №3, 143-146.

11. Карташова А. В., Аналог теоремы Маккензи для решеток топологий конечных алгебр. Фунд. прикл. матем. (2005) 11, №3, 109-117.

12. Šmarda B., The lattice of topologies of topological $l$-groups. Czech. Math. J. (1976) 26, №1, $128-136$.

13. Kartashova A. V., On lattices of topologies of unary algebras. J. Math. Sci. (2003) 114, №2, 1086-1118.

14. Курош А. Г., Теория групп. Физматлит, Москва, 1967.

15. Богомолов А. М., Салий В. Н., Алгебраические основы теории дискретных систем. Физматлит, Москва, 1997.

16. Kopeček O., A note on some cardinal functions on unary algebras. In: Contributions to General Algebra, 2 (Eigenthaler G., Kaiser H. K., Müller W. B., Nöbauer W., eds.). Teubner, Stuttgart, 1983, pp. 221-227.

17. Grätzer G., Universal algebra. Springer, Berlin, 1979.

18. Berman J., On the congruence lattices of unary algebras. Proc. Amer. Math. Soc. (1972) 36, №1, 34-38.

Статья поступила 30.10.2007.

Переработанный вариант поступил 13.12.2007. 